\title{
Communicability reveals a transition to coordinated behavior in multiplex networks
}

\author{
Ernesto Estrada ${ }^{1,2}$ and Jesús Gómez-Gardeñes ${ }^{3,4}$ \\ ${ }^{1}$ Department of Mathematics $\&$ Statistics, Institute of Complex Systems, \\ University of Strathclyde, Glasgow G1 $1 \mathrm{HX}$, UK \\ ${ }^{2}$ The Institute of Quantitative Theory and Methods, Emory University, GA 30033, USA, \\ ${ }^{3}$ Institute for Biocomputation and Physics of Complex Systems, University of Zaragoza, 50018 Zaragoza, Spain \\ ${ }^{4}$ Department of Condensed Matter Physics, University of Zaragoza, 50009 Zaragoza, Spain
}

\begin{abstract}
We analyse the flow of information in multiplex networks by means of the communicability function. First, we generalize this measure from its definition from simple graphs to multiplex networks. Then, we study its relevance for the analysis of real-world systems by studying a social multiplex where information flows using formal/informal channels and an air transportation system where the layers represent different air companies. Accordingly, the communicability, which is essential for the good performance of these complex systems, emerges at a systemic operation point in the multiplex where the performance of the layers operates in a coordinated way very differently from the state represented by a collection of unconnected networks.
\end{abstract}

PACS numbers: $89.75 . \mathrm{Hc}, 89.20 .-\mathrm{a}, 89.75 . \mathrm{Kd}$

\section{INTRODUCTION}

Complex systems have been usually considered as ensembles of entities whose interactions are encoded in the form of a complex network [1-4]. This approach neglects the fact that in real-world complex systems agents usually interact simultaneously in many diverse ways. Very recently, the study of multiplex networks has attracted a great deal of attention in the literature as diverse examples of real multiplexes have been collected and characterized [5-8]. In a multiplex, see Fig. 1, every entity of a complex system is split into $h$ layers, each representing a different kind of interaction among the agents. This kind of complex system representation is very convenient for the analysis, among others, of socio-economic and of transportation systems, where the layers represent different social communication or transportation channels.

The recent interest in multiplex networks has been mainly focused on the characterization of their structural properties [6, 8-13], the modeling of diverse dynamical processes on top of them [14-16] and the analysis of their associated critical phenomena [17-21]. The latter ones arise as a consequence of having different dynamical processes taking place simultaneously within each of the networked layers of the multiplex. Perhaps the most interesting aspect of this research is to unveil how the combination of different physical properties of each network layer yields new emergent behaviors that cannot be understood as the simple sum of the properties of each networked component. For instance, in $[16,22]$ it has been found out that multiplexes display a transition from a regime in which the system behaves as a set of independent networks to the one in which a coordinated behavior emerges. These transitions are obtained by decreasing the relative importance of the connections between the agents in each of the layers in relation to those representing the flow between the layers.
In this work, we analyze how the communication among the nodes in certain multiplex complex systems is affected by the coupling between the different layers. This analysis is carried by means of a generalization of the communicability function [23-25] to multiplex networks. The communicability function quantifies the number of possible routes that two nodes have to communicate with each other. We then show that communicability unveils the transition from a small coupling regime, when the multiplex behaves just as a collection of individual networks, to the one in which it acts in a coordinated way.

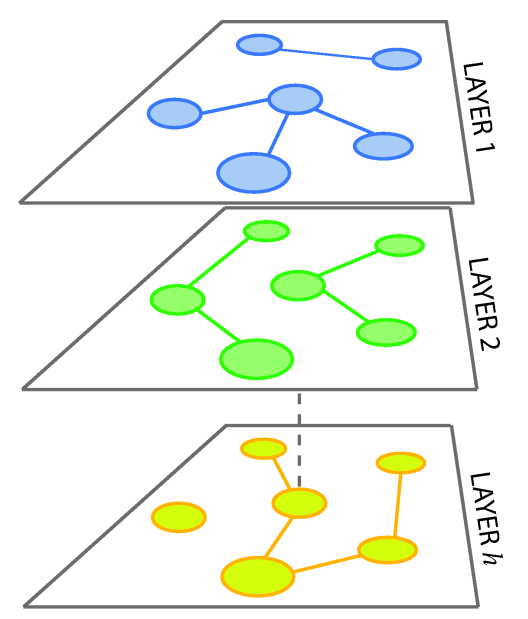

FIG. 1: Illustration of a multiplex formed by $h$ network layers. Each layer is composed of $N=6$ nodes and each of the nodes is represented in each of the layers. The connectivity of the nodes is, in principle, different in each layer of the multiplex. Apart from the connections that a node shares within each layer we consider that a node is also connected with each of its representations in the remaining network layers. 


\section{COMMUNICABILITY IN MULTIPLEXES: THEORETICAL FORMULATION}

Let us consider a multiplex formed by $h$ layers designated $L_{1}, \ldots, L_{h}$, as in Fig. 1 , and their respective $N \times N$ adjacency matrices by $\mathbf{A}_{1}, \ldots, \mathbf{A}_{h}$. The $(N \cdot h) \times(N \cdot h)$ adjacency matrix, $\mathcal{A}$, of the whole multiplex (often referred to as supra-adjacency matrix [15]) is given by:

$$
\mathcal{A}=\mathbf{A}_{L}+\mathbf{C}_{L L},
$$

where $\mathbf{A}_{L}$ is a $(N \cdot h) \times(N \cdot h)$ matrix defined as the direct sum of the adjacency matrices of the $h$ layers:

$$
\mathbf{A}_{L}=\oplus_{\alpha=1}^{h} \mathbf{A}_{\alpha}=\left(\begin{array}{llll}
\mathbf{A}_{1} & \mathbf{0} & \cdots & \mathbf{0} \\
\mathbf{0} & \mathbf{A}_{2} & \cdots & \mathbf{0} \\
\vdots & \vdots & \ddots & \vdots \\
\mathbf{0} & \mathbf{0} & \cdots & \mathbf{A}_{h}
\end{array}\right)
$$

and $\mathbf{C}_{L L}$ is a $(N \cdot h) \times(N \cdot h)$ matrix containing the inter-layer interactions:

$$
\mathbf{C}_{L L}=\left(\begin{array}{llll}
\mathbf{0} & \mathbf{C}_{12} & \cdots & \mathbf{C}_{1 h} \\
\mathbf{C}_{21} & \mathbf{0} & \cdots & \mathbf{C}_{2 h} \\
\vdots & \vdots & \ddots & \vdots \\
\mathbf{C}_{h 1} & \mathbf{C}_{h 2} & \cdots & \mathbf{0}
\end{array}\right)
$$

where $\mathbf{C}_{\alpha \beta}$ is a $N \times N$ matrix containing the interactions between nodes in layer $\alpha$ and those in layer $\beta$. Here we consider $\mathbf{C}_{\alpha \beta}=\mathbf{C}_{\alpha \beta}=\mathbf{C}=\omega I$, for all layers $\alpha$ and $\beta$, being $\omega$ a parameter describing the strength of the interlayer interactions and $\mathbf{I}$ the corresponding $N \times N$ identity matrix. With this choice, we can write:

$$
\mathbf{C}_{L L}=\mathbf{C} \otimes(\mathbf{E}-\mathbf{I}),
$$

where $\mathbf{E}$ is an all-ones $h \times h$ matrix and $\mathbf{I}$ the $h \times h$ identity matrix.

Here we are interested in accounting for all the walks between any pair of nodes in the multiplex. It is known that the number of walks of length $k$ between any pair of nodes, say $i$ and $j$, in a network is given by the $i, j$-entry of the $k$-th power of the corresponding adjacency matrix of the network. Consequently, the walks of $k$ length in a multiplex are given by the different entries of $\mathcal{A}^{k}$. In principle, the walks can contain hops of two different kinds, i.e., intra-layer and inter-layer hops. An intra-layer walk is a walk that only visits nodes and links belonging to one and only one layer $\alpha$ in the multiplex. On the other hand, inter-layer walks are those that visit nodes/links in more than one layer of the multiplex.

Following the definition of the communicability in simple networks, we are interested in giving more weight to the shortest walks than to the longer ones. Consequently, we define the $(N \cdot h) \times(N \cdot h)$ Communicability matrix of the multiplex as:

$$
\mathbf{G}=\mathbf{I}+\mathcal{A}+\frac{\mathcal{A}^{2}}{2 !}+\cdots=\sum_{k=0}^{\infty} \frac{\mathcal{A}^{k}}{k !}=\exp (\mathcal{A})
$$

Notice that when the network is weighted the different powers of the adjacency matrix, $\mathcal{A}$, still represent the number of walks of a given length between a pair of nodes. The only difference resides in the fact that now the walks are weighted in such a way that the weight of a walk is the product of the weights of all links involved in it including their repetitions.

Expressing the Communicability matrix as:

$$
\mathbf{G}=\exp (\mathcal{A})=\left(\begin{array}{llll}
\mathbf{G}_{1} & \mathbf{G}_{12} & \cdots & \mathbf{G}_{1 h} \\
\mathbf{G}_{21} & \mathbf{G}_{2} & \cdots & \mathbf{G}_{2 h} \\
\vdots & \vdots & \ddots & \vdots \\
\mathbf{G}_{h 1} & \mathbf{G}_{h 2} & \cdots & \mathbf{G}_{h}
\end{array}\right)
$$

where $\mathbf{G}_{\alpha}$ is a $N \times N$ matrix characterizing the communicability between evert pair of nodes in layer $\alpha$, we can have more insight on the information contained in G. First, let us note that $\mathbf{G}_{\alpha} \neq \exp \left(\mathbf{A}_{\alpha}\right)$ due to the coupling between the layers, i.e., $\mathbf{G}_{\alpha}$ takes into account those paths connecting two nodes $i$ and $j$ within the same layer $\alpha$ that, in principle, can include hops to any other layer $\beta \neq \alpha$. Obviously, if the inter-layer coupling is absent, $\omega=0$, all the interlayer communication is knocked out and:

$$
\mathbf{G}=\left(\begin{array}{cccc}
\exp \left(\mathbf{A}_{1}\right) & \mathbf{0} & \cdots & \mathbf{0} \\
\mathbf{0} & \exp \left(\mathbf{A}_{2}\right) & \cdots & \mathbf{0} \\
\vdots & \vdots & \ddots & \vdots \\
\mathbf{0} & \mathbf{0} & \cdots & \exp \left(\mathbf{A}_{h}\right)
\end{array}\right)
$$

so that communicability is exactly equal to that of a collection of independent networks.

In order to quantify the total amount of communicability of the nodes in the multiplex we consider both the communicability broadcasted and received, by a node $i$ in the $\alpha$-th layer of the multiplex as:

$$
\begin{aligned}
G_{\alpha}^{\mathrm{br}}(i) & =\sum_{j=1}^{N}\left(G_{\alpha}\right)_{j i}, \\
G_{\alpha}^{\mathrm{rec}}(i) & =\sum_{j=1}^{N}\left(G_{\alpha}\right)_{i j},
\end{aligned}
$$

respectively. Notice that these indices contain information about both the intra- and inter-layer walks. If all layers are symmetric, i.e. undirected networks, the broadcasted and received communicabilities of the nodes are identical: $G_{\alpha}^{\mathrm{br}}(i)=G_{\alpha}^{\mathrm{rec}}(i)$.

Finally, in order to account for the mean broadcasting and receiving activity of a node $i$ in the whole multiplex we consider the corresponding communicabilities of each of the representations of the node $i$ in each of the $h$ layers. In principle, there are different ways of averaging the effects of the distinct layers on the global communicability of a multiplex. The most common way of aggregating this information would be considering the arithmetic mean. 
In this case, if a node $i$ has a large communicability with the rest of the nodes in one layer $\alpha$ but very small in another layer $\beta$, the arithmetic mean is biased towards the highest communicability. Suppose a multiplex of 2 layers and that the maximum communicability that a node $i$ can have in a given layer is 10 . Let the communicability of this node be equal to 10 in one layer and only 0.01 in the other one. The arithmetic mean of the communicability of this node $i$ in the multiplex is 5.005, which indicates still a good global communicability. If we think however that the poor communicability between this node and the rest of nodes in the second layer is a limitation for the global communicability of that node, the arithmetic mean cannot be seen as a good way to aggregate the information among the layers. This situation is typical in the type of networks that we are describing in this work, i.e., social and transportation networks. For instance, if two individuals have large communicability in a formal layer of communication but very poor one in an informal one, the global communicability will be limited by the smallest communicability occurring in one of the layers. This is equivalent to say that we can consider that the communication is flowing among the nodes in a parallel way. In this case the use of the harmonic mean, defined as:

$$
H(i)^{\text {type }}=\frac{h}{\sum_{\alpha=1}^{h} G_{\alpha}^{\text {type }}(i)^{-1}},
$$

(type $=$ br, rec), is more appropriate than the arithmetic one. The harmonic mean of the communicability for the hypothetical node $i$ that we described before is about 0.02 , which remark the fact that the communicability in one of the layers is very small thus making its global communicability to be very poor. Thus, hereafter we will refer only the harmonic mean $H^{\text {type }}(i)$ of the communicability (broadcasted or received) by this node in all the layers of the multiplex [26]. Finally, in order to compare the results we use the aggregate network $\hat{\mathcal{G}}$ defined as follows. Let $\mathcal{G}_{1}=\left(V, E_{1}\right), \mathcal{G}_{2}=\left(V, E_{h}\right), \ldots, \mathcal{G}_{h}=\left(V, E_{h}\right)$ be the set of network layers of the multiplex. Then, $\hat{\mathcal{G}}=(V, \hat{E})$ where $\hat{E}=\cup_{\alpha=1}^{h} E_{\alpha}$.

\section{COMMUNICABILITY IN MULTIPLEXES: APPLICATION TO REAL SYSTEMS}

In this section we will apply the formulation developed above to the analysis of two real complex systems whose structure is described by a multiplex, namely, a social and a transportation multiplexes. Our idea is to capture how the coupling between layers acts on the communicability properties of these systems, being it fundamental for their respective functioning.
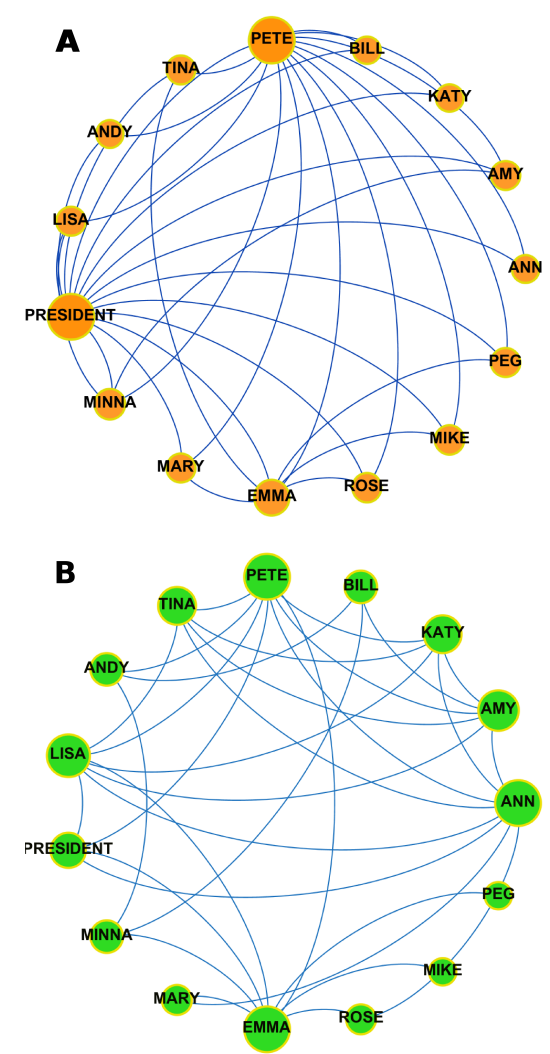

FIG. 2: We show the formal (A) and informal (B) communication layers among the 15 members of the organization studied in [27]. The formal layer of communication forms a directed network while the informal one, representing friendship ties, is undirected.

\section{Communicability in a social multiplex}

In most socio-economical organizations there is a formal or official structure, which defines the official hierarchy, lines of authority and of communication. In parallel, there is a network of friendships that tie people together in ways that have nothing to do with the official structure. This situation is very clear in a social multiplex obtained as the result of 16 months of observation of an office politics [27]. The office is formed by 15 members of an overseas branch of a large international organization. This multiplex is formed by two layers, the first layer corresponds to a directed network comprising the formal organizational chart of the employees, whereas the second layer represents the informal association among the employees. The two layers of the multiplex network are represented in Fig. 2.

During the former period two employees, Emma and Minna, were the targets of a leveling coalition formed by 6 members of staff. From a network perspective the identification of the attacking coalition is not difficult as their members form a clique in the informal social layer 


\begin{tabular}{lccc}
\hline & Formal & Informal & Aggregate \\
\hline PETE & 17.50 & 436.52 & 1137.76 \\
ANN & 1.00 & 414.39 & 873.45 \\
AMY & 1.00 & 353.47 & 682.67 \\
KATY & 1.00 & 337.97 & 652.99 \\
TINA & 1.00 & 337.97 & 652.99 \\
LISA & 1.00 & 419.20 & 909.02 \\
EMMA & 6.00 & 274.51 & 776.81 \\
\hline MINNA & 3.00 & 88.97 & 442.81 \\
\hline PRESIDENT & 26.17 & 268.40 & 1137.76 \\
BILL & 1.00 & 99.35 & 218.13 \\
ANDY & 1.00 & 111.75 & 279.47 \\
MARY & 1.00 & 121.19 & 254.66 \\
ROSE & 1.00 & 121.19 & 254.66 \\
MIKE & 1.00 & 49.94 & 120.99 \\
PEG & 1.00 & 49.94 & 120.99
\end{tabular}

TABLE I: Broadcasted communicability in the formal and informal layer of communication for the 15 members of the social multiplex. We show the case of zero coupling between the two layers $(\omega=0)$ as well as the case of the aggregate network.

of the multiplex. This coalition is formed by Ann, Katy, Amy, Pete, Tina and Lisa. The analysis of the communicability in the informal layer of the multiplex also reveals the importance of this coalition in the diffusion of information in the network. In Table 1 it can be seen that the six members of the coalition are the highest broadcasters of information in this layer in agreement with the observation made by Thurman that [22]: "Within the network a large number of rumors circulated rapidly among Pete, Ann, Amy, Katy, Tina, and Lisa." However, nothing is evident about the victims of the attack from the analysis of the separated layers. In the informal layer of communication, Emma occupies the position immediately after the attacking coalition in the ranking of broadcasted communicability. However, Minna only appears at the bottom three of the ranking together with Mike and Peg. In the formal layer there are only four broadcasters: Pete, the President, Emma and Minna. We recall that Emma had been promoted to administrative manager and Minna was also in a managerial position. However, neither the communicability at the formal nor at the informal layer reveals any hint about the plausible causes for the attacks. On the other hand, in the aggregate network the ranking of the employees according to their broadcasted communicability is mixed up and while Emma is the fifth in broadcasting information, Minna occupies the position number nine.

The communicability matrix $\mathbf{G}$ containing the flow of information between every pair of employees in the office for the two layers (administrative or formal and informal) is given in Fig. 3 for two different values of the strength

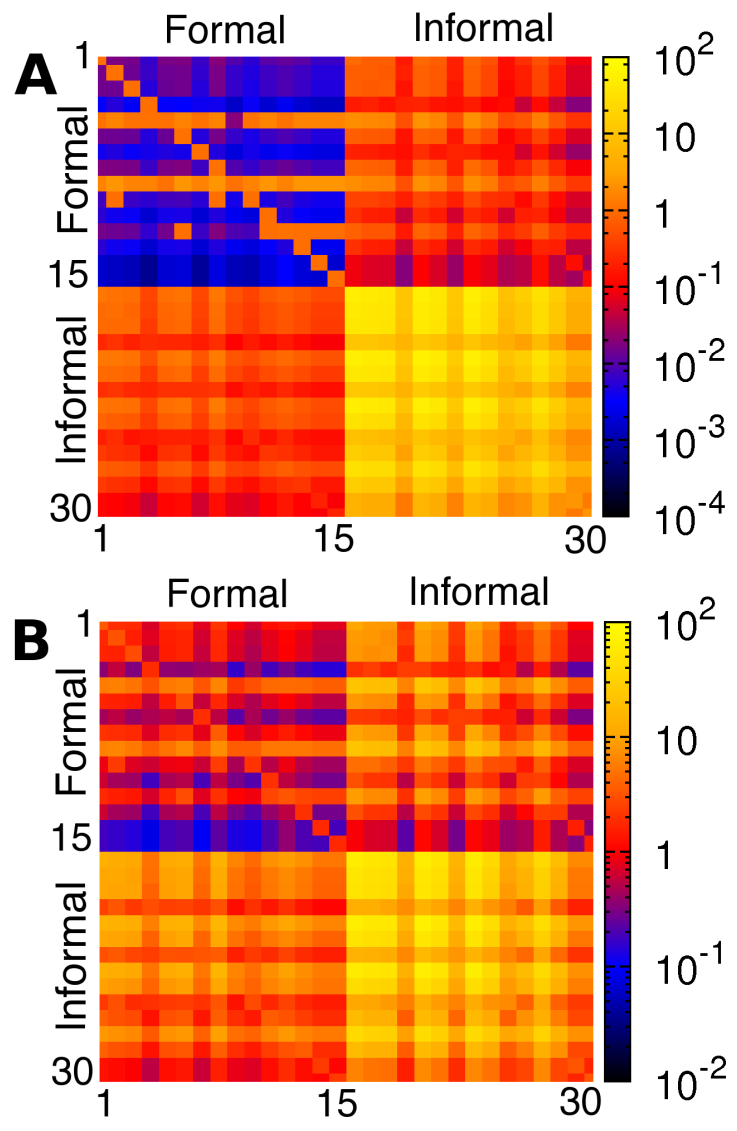

FIG. 3: We show two contour plots of the communicability between the 15 members for two different coupling constants between the formal and informal layers of communication: $\omega=0.1(\mathrm{~A})$ and $\omega=1(\mathrm{~B})$. The indexes of the nodes in the Formal layer correspond to: 1: ANN, 2: AMY, 3: KATY, 4: BILL, 5: PETE, 6: TINA, 7: ANDY, 8: LISA, 9: PRESIDENT, 10: MINNA, 11: MARY, 12: EMMA, 13: ROSE, 14: MIKE, 15: PEG. The nodes labeled from 16 to 30 are just the corresponding representations of the same actors (in the same former order) in the Informal layer.

of the interlayer interaction $\omega=0.1$ (panel A) and $\omega=1$ (panel B). From these two matrices it can be seen that most of the communication flow takes place on the informal layer of the multiplex.

We consider now the harmonic mean of the communicability broadcasted in both layers for different values of the coupling constant (see Table 2). When the coupling between the formal and informal layers is relatively weak $(0.1 \leq \omega<0.5)$ Emma and Minna occupy a privileged position in their broadcasting communicability, which place them only after Pete and the President and well over the rest of the members of the attacking coalition, who at the same time are better broadcasters than the rest of the employees. As the coupling constant $\omega$ increases the informal communication layer re- 


\begin{tabular}{lccccc}
\hline \multicolumn{7}{c}{$\omega=0.1$} & $\omega=0.3$ & $\omega=0.5$ & $\omega=0.8$ & $\omega=1.0$ \\
\hline PETE & 34.47 & 41.01 & 54.13 & 86.51 & 117.06 \\
ANN & 2.36 & 5.30 & 11.27 & 26.27 & 40.73 \\
AMY & 2.30 & 4.71 & 9.61 & 21.92 & 33.78 \\
KATY & 2.28 & 4.58 & 9.25 & 20.98 & 32.29 \\
TINA & 2.28 & 4.58 & 9.25 & 20.98 & 32.29 \\
LISA & 2.37 & 5.38 & 11.49 & 26.85 & 41.67 \\
\hline EMMA & 12.12 & 15.16 & 21.28 & 36.56 & 51.15 \\
MINNA & 6.00 & 7.55 & 10.66 & 18.29 & 25.44 \\
\hline PRESIDENT & 48.44 & 54.47 & 66.51 & 95.88 & 123.32 \\
BILL & 2.07 & 2.83 & 4.35 & 8.18 & 11.86 \\
ANDY & 2.10 & 3.02 & 4.88 & 9.58 & 14.10 \\
MARY & 2.10 & 3.01 & 4.87 & 9.53 & 14.01 \\
ROSE & 2.10 & 3.01 & 4.87 & 9.53 & 14.01 \\
MIKE & 2.02 & 2.47 & 3.39 & 5.69 & 7.91 \\
PEG & 2.02 & 2.47 & 3.39 & 5.69 & 7.91
\end{tabular}

TABLE II: Harmonic means of the broadcasted communicability for the 15 members of the social multiplex studied for different values of the coupling constant $\omega$.

ceives more importance in determining the amount of information broadcasted. In this scenario, Minna starts to loss their hierarchy in broadcasting information and she passes from being the 4 th broadcaster at $0.1 \leq \omega<0.5$ to the 9 th place for $\omega \geq 0.7$. This situation might explain why Emma and Minna have been the object of the coalition attacks. The coalition, which is very well communicated at the informal level, could see in Emma and Minna as a thread to their position as major broadcasters or controllers of the information flow in the office. This, of course, would never happen if the employees consider the informal level of communication only. But the coupled communication between the two layers, which the actors of the network would perceive as a unique block in which the information is propagated, well justify the feeling of this thread. This example clearly illustrates how neither the isolated layers nor the aggregate network can explain the ways in which information flows in a multiplex and affects its nodes.

\section{Communicability in an airports multiplex}

Information, generally speaking, not only flows across the multiple layers of social systems. Airport transportation networks also represent an excellent example of a coupled multiplex system. Here we consider 450 European airports and 6 airlines, subdivided into major or traditional (British Airways, Lufthansa and AirFrance) and low-cost fares (Easyjet, AirBerlin and Ryanair). Each layer represents the air connectivity between the 450 airports provided by the corresponding airline [6]. The networks in each layer are undirected as if there is a flight from airport A to airport B, there is always a returning flight from B to A.

Our main goal here is to study how airport centrality, in terms of communicability, emerges from the coupling between the layers in the multiplex. We start by studying the harmonic mean, $H(i)$, of the communicabilities, $G_{\alpha}(i)$, of airport $i$ in each of the layers $\alpha$ for different values of the coupling constant $\omega$. When there is no coupling between the layers, i.e., $\omega=0, H(i)$ represents the harmonic mean of the communicabilities of $i$ in each isolated layer or airline. As it can be seen in Table III these airports are mainly the bases for major airline companies, such as Paris Charles de Gaulle (AirFrance) or those with the presence of most of the six airlines studied. In fact, if we consider the harmonic mean for the communicabilities of each airport $i$ averaging over only those (3) layers corresponding to major airlines, $H^{\text {major }}(i)$, or, respectively, over those (3) layers representing low-cost companies, $H^{\text {low-cost }}(i)$, companies, we observe that the communicability in the uncoupled networks is dominated by major companies. For instance, the Pearson correlation coefficient between $H(i)$ and $H^{\text {major }}(i)$ is 0.76 , while that for $H(i)$ and $H^{\text {low-cost }}(i)$ is only 0.29 .

As soon as some coupling between the layers is allowed a different picture starts to emerge. For a small coupling constant, such as $\omega=0.1$, a few new airports show up as the most central ones in terms of their communicability. For instance, the London Stansted and Dublin airports now appear among the top ten most central airports in terms of their communicability. These airports are the main bases for low-cost fare companies such as Ryanair. Among the companies studied, Ryanair also has the largest presence in the airport of Madrid Barajas, which now occupies the second place in the ranking. When the coupling between the layers in the multiplex increases further, such as to $\omega=1.0$, these three latter airports become the most central ones. However, this increment in the relevance of these airports with heavy presence of low-cost companies is not developed in detriment of the role played by major airlines.

If we consider the correlation coefficient between $H(i)$ and $H^{\text {major }}(i)$ for the coupling $\omega=0.1$ it is 0.97 and that for $H(i)$ vs. $H^{\text {low-cost }}(i)$ has also increased up to 0.65 . For the coupling constant $\omega=1.0$ these correlations have increased to 0.99 and 0.98 , respectively. In Fig. 4 we show the scatter plots $H^{\text {type }}(i)$ (type: major and low-cost) versus $H(i)$ for $\omega=0.1$ and 1.0, show the increase of correlations as the inter-layer coupling $\omega$ grows. As a consequence, the increase in the coupling between the different layers in the multiplex equilibrates the role played by major and low-cost companies in determining the centrality of the respective airports. In other words, by coupling with a moderate strength the airlines of the multiplex a situation in which major and low-cost airlines operate in a coordinated way shows up. However, the coupling for this balanced regime has to be moderate 


\begin{tabular}{clll}
\hline Rank $\omega=0.0$ & $\omega=0.1$ & $\omega=1.0$ & Aggregate \\
\hline 1 Paris CdG & London Stansted & London Stansted & Frankfurt \\
2 Barcelona & Madrid & Dublin & Munich \\
3 Venice & Barcelona & Madrid & London Stansted \\
4 Amsterdam & Paris CdG & Palma de Mallorca & London Gatwick \\
5 Copenhagen & Dublin & Bergamo & Larnaca \\
6 Madrid & Malaga & Alicante & Düsseldorf \\
7 Frankfurt & Bergamo & Barcelona & Madrid \\
8 Prague & Palma de Mallorca Malaga & Paris CdG \\
9 Athens & Venice & Brussels South & Palma de Mallorca \\
10 Tolouse-Blagnac & Alicante & Pisa & Barcelona
\end{tabular}

TABLE III: Ranking of European airports on the basis of their harmonic mean of communicability for different coupling constants in the multiplex and for the aggregate network.

enough since increasing more $\omega$ we approach the aggregate network. In this case the correlation coefficients between $H(i)$ and $H^{\text {major }}(i)$ and $H(i)$ and $H^{\text {low-cost }}(i)$ have dropped to 0.7 and 0.87 pointing out a less equilibrated regime than that for moderate values for inter-layer coupling $\omega$.
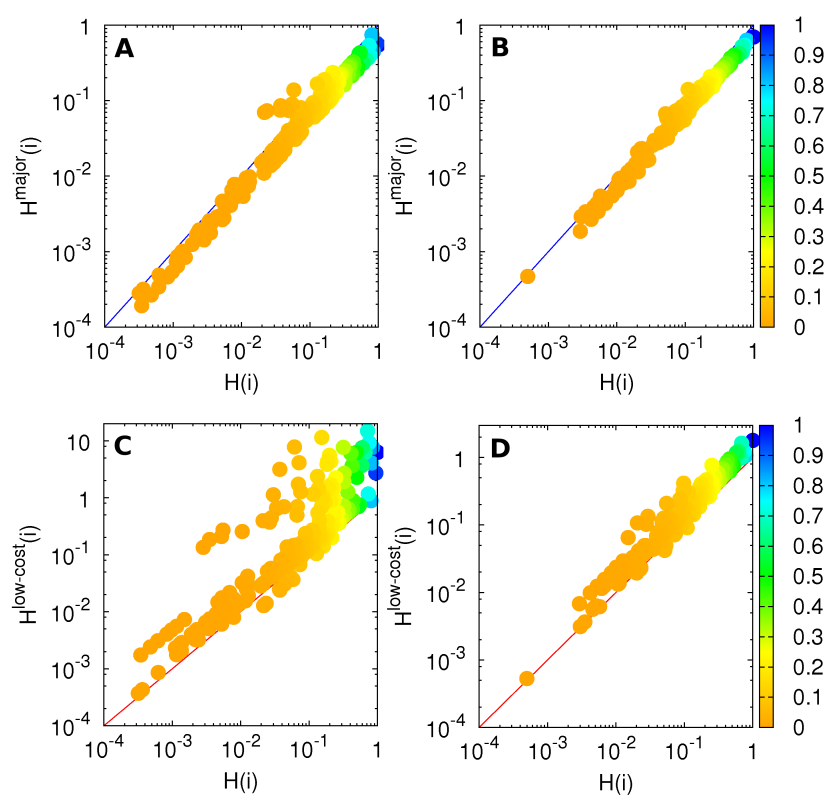

FIG. 4: We show the scatter plots $H^{\text {major }}(i)$ versus $H(i)$ (panels A and B) and those $H^{\text {low-cost }}(i)$ versus $H(i)$ (panels $\mathrm{C}$ and $\mathrm{D})$. The left panels (A and $\mathrm{C}$ ) are for inter-layer coupling $\omega=0.1$ whereas those in the right ( $\mathrm{B}$ and $\mathrm{D})$ are for $\omega=1$ : The increase of the coupling yields an increase of the correlation between the harmonic mean of the communicabilities of a node $i$ across all the layers and that considering only those specific (major and low-cost) ones. The color bar in the right represent in linear scale the value of $H(i)$.

\section{CONCLUSIONS}

In this work we have analyzed the flow of information in multiplex networks by means of their communicability. After generalizing this measure from the case of simple networks to the most realistic scenario of multiplex networks, we have studied its relevance in two real systems. The first represents a small social multiplex formed by individuals in an organization, in which the information flows across a formal layer reflecting the hierarchical structure of the organization and another one representing the informal ways of communication among the actors. The second multiplex represents the European Air-transportation system in which air traffic between European airports is operated by 6 air-companies. Our study points out that the communicability, being essential for the good performance of these two real systems, shows the difference between a collection of unconnected networks and the systemic operation point in which the performance of the layers operates in coordinated way. In both cases the multiplex nature of the systems is essential to explain the flow of information and the centrality of nodes different to the simplistic limits in which the networked layers are disconnected or aggregated.

This work has been partially supported by the Spanish MINECO under projects FIS2011-25167 and FIS201238266-C02-01; and by the European FET project MULTIPLEX (317532. J.G.G. is supported by the MINECO through the Ramón y Cajal Program.

[1] R. Albert, and A.-L. Barabási, Rev. Mod. Phys. 74, 47 (2002).

[2] M.E.J. Newman, SIAM Rev. 45, 167 (2003).

[3] S. Boccaletti, V. Latora, Y. Moreno, M. Chavez and, D.U. Hwang, Phys. Rep. 424, 175 (2006). 
[4] S.N. Dorogovtsev, A.V. Goltsev, and J.F.F. Mendes, Rev Mod. Phys. 80, 1275 (2008).

[5] M. Kurant and P. Thiran, Phys. Rev. Lett. 96, 138701 (2006).

[6] A. Cardillo, J. Gómez-Gardeñes, M. Zanin, M. Romance, D. Papo, F. del Pozo, and S. Boccaletti, Scientific Reports 3, 1344 (2013).

[7] M. Szell, R. Lambiotte, and S. Thurner, Proc. Natl Acad. Sci. USA 107, 1003.5137 (2010)

[8] P. J. Mucha, T. Richardson, K. Macon, M. A. Porter, and J.-P. Onnela, Science 328, 876 (2010).

[9] A. Sole-Ribalta, M. De Domenico, N.E. Kouvaris, A. Diaz-Guilera, S. Gómez, A. Arenas, Phys. Rev. E 88, 032807 (2013).

[10] M. De Domenico, A. Solé-Ribalta, E. Cozzo, M. Kivela, Y. Moreno, M.A. Porter, S. Gómez, and A. Arenas, Phys. Rev. X (in press).

[11] V. Nicosia, G. Bianconi, V. Latora, and M. Barthelemy, Phys. Rev. Lett. 111, 058701 (2013).

[12] M. De Domenico, A. Soé-Ribalta, E. Omodei, S. Gómez, and A. Arenas, arXiv:1311.2906.

[13] E. Cozzo, M. Kivela, M. De Domenico, A. Solé, A. Arenas, S. Gómez, M.A. Porter, and Y. Moreno, arXiv:1307.6780.

[14] J. Gómez-Gardeñes, I. Reinares, A. Arenas, and L. M. M. Floría, Scientific Reports 2, 320 (2012).

[15] M. De Domenico, A. Sole, S. Gómez, and A. Arenas,
arXiv:1306.0519.

[16] S. Gómez, A. Díaz-Guilera, J. Gómez-Gardeñes, C. J. P. Pérez-Vicente, Y. Moreno, and A. Arenas, Phys. Rev. Lett. 110, 028701 (2013).

[17] G. J. Baxter, S. N. Dorogovtsev, A. V. Goltsev, and J. F. F. Mendes, Phys. Rev. Lett. 109, 248701 (2012).

[18] E. Cozzo, A. Arenas, and Y. Moreno, Phys. Rev. E 86, 036115 (2012)

[19] G. Bianconi, Phys. Rev. E 87, 062806 (2013).

[20] C. Granell, S. Gómez, and A. Arenas, Phys. Rev. Lett. 111, 128701 (2013).

[21] E. Cozzo, R.A. Baños, S. Meloni, and Y. Moreno, Phys. Rev. E 88, 050801(R) (2013).

[22] F. Radicchi, and A. Arenas, Nature Physics 9, 717 (2013).

[23] E. Estrada, and N. Hatano, Phys. Rev. E 77, 036111 (2008).

[24] E. Estrada, and D. Higham, SIAM Rev. 52, 696 (2010).

[25] E. Estrada, N. Hatano, and M. Benzi, Phys. Rep. 514, 89 (2012).

[26] We have tested both types of means (arithmetic and harmonic) and we have found empirical evidences that support the use of the harmonic over the arithmetic mean. The data is not shown but it can be obtained by request to the corresponding author.

[27] B. Thurman, Social Networks 2, 47 (1979). 